\title{
Gemcitabin: Geeignete Basis für Kombinationen mit neuen Substanzen
}

\begin{abstract}
Als Standard in der First-line-Therapie des inoperablen nicht-kleinzelligen Bronchialkarzinoms (NSCLC) gelten platinhaltige Dubletten [1]. Besonders überzeugend ist die Datenlage aufgrund der Vielzahl an Studien für Gemcitabin (Gemzar $\left.{ }^{\circledR}\right) /$ Platin-Regime [2]. Diese Kombination stellt demnach ein Fundament für die Kombinationstherapie gerade mit neuen Substanzen dar. Auf dem diesjährigen ASCO-Kongress wurden Daten zur Kombination von Gemcitabin/Platin mit jeweils zwei Antikörpern vorgestellt. Neben Gemcitabin ist Pemetrexed (Alimta ${ }^{\circledR}$ ) ein möglicher neuer Kombinationspartner in der First-line-Therapie: Das Zytostatikum, bislang Standard bei vorbehandelten NSCLC-Patienten, lässt sich gut mit Carboplatin kombinieren und ist bei geringerer Toxizität ebenso aktiv wie Gemcitabin/Carboplatin.
\end{abstract}

Leitliniengemäß sollten bei NSCLC-Patienten im Stadium IIIB/IV Zweierkombinationen eingesetzt werden [3]. Zytostatische Dreierkombinationen haben sich bisher aufgrund einer erhöhten Toxizität ohne gesteigerte Effektivität nicht bewährt. Aktuelle Studien, die beim diesjährigen ASCO vorgestellt wurden, belegen, dass mit der richtigen Basis auch Dreierkombinationen einen Vorteil für den Patienten bringen können. Wichtige Voraussetzung einer solchen Basiskombination ist eine starke Wirksamkeit bei gleichzeitig günstigem Toxizitätsprofil, wie es bei Gemcitabin/ Platin-Regimen bereits in zahlreichen Studien gezeigt werden konnte. Neue PhaseIII-Studien konnten belegen, dass sich die in Europa beim NSCLC am häufigsten eingesetzte Dublette Gemcitabin/Cisplatin (GC) auch mit neuen gegen VEGF (vascular endothelial growth factor) oder EGFR (epidermal growth factor receptor) gerichteten Antikörpern gut kombinieren lässt $[4,5]$.

\section{Gemcitabin/Platin kombiniert mit VEGF-Antikörper}

In einer internationalen Studie wurde eine Kombination aus Gemcitabin/Platin und dem VEGF-Antikörper Bevacizumab untersucht [4]. 1043 nicht vorbehandelte NSCLC-Patienten in inoperablem Stadium IIIB-IV und einem ECOG Performancestatus von $0-1$ wurden randomisiert. Aus Toxizitätsgründen wurden unter anderem Patienten mit Plattenepithelkarzinomen, mit einer Hämoptyse $\geq 2$, mit Hirntumoren oder mit unkontrolliertem Bluthochdruck ausgeschlossen. Die Patienten wurden mit Gemcitabin $1250 \mathrm{mg} / \mathrm{m}^{2}$, Cisplatin $80 \mathrm{mg} / \mathrm{m}^{2}$ und Bevacizumab (7,5 oder $15 \mathrm{mg} / \mathrm{kg}$ ) oder Plazebo behan- delt. Primärer Endpunkt war das progressionsfreie Überleben. Als sekundäre Endpunkte waren Gesamt-Überleben, Responseraten und -dauer sowie Sicherheit definiert.

In allen 3 Armen wurden median 5-6 Zyklen der Kombinationstherapie verabreicht. Durch die Dreierkombination mit der Gemcitabin/Platin-Basis plus Bevacizumab konnte der primäre Endpunkt signifikant verbessert werden, berichtete Christian Manegold aus Mannheim: Bei Zugabe von Bevacizumab $\left(7,5 \mathrm{mg} / \mathrm{m}^{2}\right)$ zum Gemcitabin/Platin-Regime verlängerte sich die mediane progressionsfreie Überlebenszeit auf 6,7 Monate (Tab. 1), mit der höheren Dosierung von $15 \mathrm{mg} / \mathrm{m}^{2}$ auf 6,5 Monate, wobei auch ohne Zugabe des Antikörpers eine sehr hohe Basis von 6,1 Monaten erreicht wurde. Es konnte ein Anstieg der Responserate von 20\% auf $34 \%\left(7,5 \mathrm{mg} / \mathrm{m}^{2}\right)$ bzw. auf $30 \%$ $\left(15 \mathrm{mg} / \mathrm{m}^{2}\right)$ erzielt werden. Die Daten zum Gesamtüberleben sind noch nicht vorhanden.

Charakteristische Toxizitäten für Bevacizumab sind Hypertonie, Proteinurie, ischämische Ereignisse, Thromboembolien und pulmonale Blutungen.

\begin{tabular}{|c|c|c|c|}
\hline & $\begin{array}{l}\text { Gemzar/Cisplatin } \\
+ \text { Plazebo } \\
(\mathrm{n}=347)\end{array}$ & $\begin{array}{l}\text { Gemzar/Cisplatin + } \\
\text { Bevacizumab } \\
7,5 \mathrm{mg} / \mathrm{kg}(\mathrm{n}=345)\end{array}$ & $\begin{array}{l}\text { Gemzar/Cisplatin + } \\
\text { Bevacizumab } \\
15 \mathrm{mg} / \mathrm{kg}(\mathrm{n}=351)\end{array}$ \\
\hline \multicolumn{4}{|c|}{ Progressionsfreies Überleben } \\
\hline Median (Monate) & 6,1 & 6,7 & 6,5 \\
\hline Responserate, $\%$ & 20 & 34 & 30 \\
\hline \multicolumn{4}{|l|}{ Responsedauer } \\
\hline Median (Monate) & 4,7 & 6,1 & 6,1 \\
\hline $95 \%$-CI & 4,$6 ; 5,6$ & 5,$1 ; 7,0$ & 5,$0 ; 6,6$ \\
\hline
\end{tabular}

Tab. 1. Höhere progressionsfreie Überlebens- und Ansprechraten sowie längere Ansprechdauer bei Kombination von Gemcitabin/Cisplatin mit Bevacizumab.

\section{Gemcitabin/Platin mit EGFR- Blockade kombinieren}

Für eine weitere Kombination mit Gemcitabin/ Platin als Basis wurden viel versprechende Daten vorgestellt. Butts et al. verglichen in einer randomisierten Phase-II-Studie bei 131 nicht vorbehandelten NSCLC-Patienten die Dubletten Gemcitabin/Cisplatin oder Gemcitabin/Carboplatin (Arm B) mit einer Dreierkombination [5]. Im Arm A wurde das Gemcitabin/Platin-Regime mit dem gegen EGFR gerichteten Antikörper Cetuximab kombiniert. Höhere Responseraten sowie längeres progressionsfreies und Gesamt-Überleben sprechen für den Nutzen der Gemcitabin/Platin-Basis in Kombination mit Cetuximab. Auf die Chemotherapie sprachen 18,2\% der Patienten an; bei zusätzlicher AntikörperGabe stieg die Responserate auf $27,7 \%$. Das progressionsfreie Überleben war mit 5,1 Monaten in Arm A länger als bei alleiniger Chemotherapie mit 4,2 Monaten. Unter einer Kombination aus Gemcitabin/Platin und Cetuximab lag die Gesamt-Überlebenszeit bei 11,9 versus 9,3 Monaten ohne Hinzugabe des EGFR-Antikörpers. Die Hämatotoxizität ( Grad 3/4) war in beiden Armen etwa vergleichbar; Haut- und Infusionsreaktionen 
Tab. 2. Das Regime Pemetrexed/Carboplatin besitzt bei gleicher Effektivität eine bessere Verträglichkeit als Gemcitabin/ Carboplatin.

\begin{tabular}{|c|c|c|c|c|c|}
\hline & \multicolumn{2}{|c|}{ Pemetrexed/Carboplatin $(\mathrm{n}=212)$} & \multicolumn{2}{|c|}{ Gemcitabin/Carboplatin $(\mathrm{n}=210)$} & \multirow[b]{2}{*}{$\mathrm{p}$} \\
\hline & Grad 3, \% & $\operatorname{Grad} 4, \%$ & Grad 3, \% & Grad 4, \% & \\
\hline Anämie & 11 & 1 & 12 & 1 & N3 \\
\hline Leukopenie & 17 & 5 & 35 & 9 & $<0,001$ \\
\hline Granulozytopenie & 24 & 14 & 25 & 23 & 0,03 \\
\hline Thrombozytopenie & 13 & 11 & 31 & 23 & $<0,001$ \\
\hline Bluttransfusion & \multicolumn{2}{|c|}{28} & \multicolumn{2}{|c|}{42} & 0,003 \\
\hline Plättchentransfusion & \multicolumn{2}{|c|}{3} & \multicolumn{2}{|c|}{9} & 0,007 \\
\hline $\begin{array}{l}\text { Todesfälle aufgrund } \\
\text { neutropener Infektionen }\end{array}$ & \multicolumn{2}{|c|}{$\mathrm{n}=2$} & \multicolumn{2}{|c|}{$\mathrm{n}=3$} & \\
\hline
\end{tabular}

waren mit Cetuximab jedoch häufiger. Die Toxizität ist handhabbar, schlussfolgern die Autoren.

\section{Gute Evidenz der Gemcitabin/Platin- Kombinationen}

Grundsätzlich können Gemcitabin/PlatinKombinationen zu den effektivsten Regimen in der First-line-Therapie des NSCLC gezählt werden. Dafür sprechen Daten von 51 Phase-III-Studien sowie eine umfangreiche Metaanalyse von insgesamt 13 Studien. Letztere zeigte einen signifikanten Vorteil für Gemcitabin/Platin-Regime bei progressionsfreiem und Gesamt-Überleben im Vergleich zu anderen platinbasierten Kombinationen [2].

\section{Pemetrexed - neue Option für die First-line-Therapie}

In der Second-line-Therapie des NSCLC hat sich Pemetrexed (Alimta ${ }^{\circledR}$ ) als effektives und gut verträgliches Zytostatikum bewährt. Basierend auf den Daten einer PhaseIII-Studie versus Docetaxel gilt die Subs-

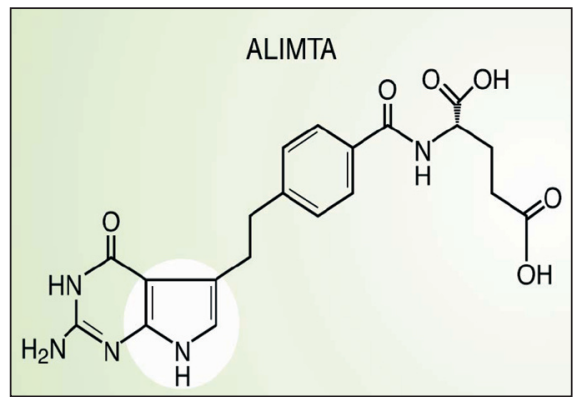

Abb. 1. Wirkgrafik Alimta ${ }^{\circledR}$ $\operatorname{tanz}$ wegen ihrer ebenso hohen Effektivität bei erheblich besserem Toxizitätsprofil heute als Mittel der Wahl bei vortherapierten Patienten [6]. Eine Phase-III-Studie der «Norwegian Lung Cancer Group» zeigt deutlich, dass Pemetrexed zukünftig auch in der First-line-Therapie eine attraktive Option sein kann [7]. Bereits in Phase-II-Studien hatte sich Pemetrexed/Platin als ebenso effektiv wie Standard-Dubletten erwiesen, zeigte jedoch ein günstigeres Toxizitätsprofil [8, 9]. Für die von Björn H. Grönberg, Trondheim, vorgestellte Studie wurden 446 therapienaive NSCLC-Patienten $\mathrm{zu}$ einer Therapie mit dem Standard Gemcitabin/ Carboplatin oder mit Pemetrexed/Carboplatin randomisiert.

\section{Pemetrexed dem Standard ebenbürtig}

Das Pemetrexed/Platin-Regime konnte mit Effektivität und geringerer Toxizität überzeugen. Die Wirksamkeit des Pemetrexed/ Platin-Regimes zeigte sich durch eine $\mathrm{Ge}$ samt-Überlebenszeit von 7,3 versus 7 Monaten im Gemcitabin/Platin-Arm. Dabei war die Hämatotoxizität mit Pemetrexed/Platin signifikant niedriger (Tab. 2). Auch Bluttransfusionen (28 versus $42 \%$ ) und Thrombozytenkonzentrate wurden signifikant seltener benötigt, als mit Gemcitabin/Carboplatin.

Durch das in dieser Studie gezeigte, vorteilhafte Nutzen/Risiko-Profil empfiehlt sich die Pemetrexed/Carboplatin-Kombination auch für NSCLC- Patienten mit schlechtem Performance-Status und Komorbidität.

Aufgrund einer weiteren Phase-III-Studie mit Pemetrexed/Cisplatin versus Gemcitabin/Cisplatin in der First-line-Therapie des NSCLC wurde die Zulassung für diese Indikation beantragt [10].

\section{Referenzen}

1 Le Chevalier et al.: Br Med J $199 ; 331: 903$.

2 Le Chevalier et al.: Lung Cancer 2005;47:69-80. 3 Interdisziplinäre Leitlinie der Deutschen Krebsgesellschaft : Therapie des Bromnchialkarzinoms; http://www.uni-duesseldorf.de/awmf/ll-na/032007.htm

4 Manegold C, et al.: J Clin Oncol 2007; ASCO Ann Meet Proc 25, No. 18S: Abstr \#LBA7514. 5 Butts et al.: J Clin Oncol 2007; ASCO AAnnMeet Proc25, No. 18S: Abstr \#7539.

6 Hanna et. al.: J Clin Oncol 2004; 22:1589-1597. 7 Grönberg BH, et al.: J Clin Oncol 2007; ASCO Ann Meet Proc 25, No. 18S: Abstr \#7517.

8 Scagliotti GV, Kortsik C, Dark GG, et al.: Clin Cancer Res 2005;11(2 Pt 1):690-696.

9 Zinner RG, Fossella FV, Gladish GW, et al: Cancer. 2005;104:2449-2456.

10 Scagliotti et al.: JTO August 2007, 12th World Conference on Lung Cancer, 2(S 4):S306.

\section{Quelle}

ASCO Annual Meeting, Chicago, IL, USA, 1.-5. Juni 2007.

\section{Bericht}

Dr. Katharina Arnheim, Berlin

\section{Impressum}

Aktuelles zum NSCLC vom ASCO 2007 Gemcitabin: Geeignete Basis für Kombinationen mit neuen Substanzen

PharmaForum in ONKOLOGIE 30 | 12 | 07

PM 040622

(c) 2007 by S. Karger Verlag für Medizin und Naturwissenschaften $\mathrm{GmbH}$

Lörracher Straße 16a

D-79115 Freiburg

Tel. +49-761-45-20-70

E-mail Information@Karger.de

Mit freundlicher Unterstützung von Lilly Deutschland GmbH. 